\title{
Caracterização acústica de vogais orais na fala infantil: o falar florianopolitano*
}

\author{
Acoustic characterization of oral vowels on children speech: the dialect from Florianopolis \\ Lilian Elisa Minikel Brod \\ Izabel Christine Seara \\ Universidade Federal de Santa Catarina - Florianópolis - Santa Catarina - Brasil
}

\begin{abstract}
Resumo: Este artigo teve como objetivo apresentar uma descrição acústica de vogais orais tônicas do português brasileiro produzidas por crianças florianopolitanas de 10 e 11 anos de idade, a partir dos parâmetros de duração relativa e primeiro e segundo formantes. Para evitar os efeitos das diferenças fisiológicas entre crianças do sexo masculino e feminino, os dados foram normalizados a partir do método Lobanov. Duas análises foram realizadas. Primeiro, os dados normalizados das sete vogais orais foram analisados em função do gênero e os resultados mostraram diferenças significativas para a vogal baixa, sendo mais centralizada para crianças do sexo masculino. Segundo, os dados normalizados das vogais [e, a, o] foram analisados em função da tonicidade e ponto de articulação. Foram observadas diferenças significativas em relação à tonicidade e ponto de articulação para ambos os grupos analisados.
\end{abstract}

Palavras-chave: Vogais orais; Falar florianopolitano; Fala infantil; Descrição acústica

\begin{abstract}
The aim of this study was to present an acoustic analysis of Brazilian Portuguese (BP) stressed oral vowels produced by children aged 10 and 11 years old, regarding F1, F2 and relative duration. In order to avoid physiological differences between male and female, data were normalized using Lobanov method. Two analyses were performed. First, normalized data of the seven oral vowels were analyzed as a function of gender. Results showed significant differences for the low vowel regarding to gender, being more centralized for male subjects. Second, due to the sample size, normalized data of the vowels $[e, a, o]$ were analyzed as a function of stress and place of articulation. Significant differences were found regarding to stress and place of articulation for both groups, male and female.
\end{abstract}

Keywords: Oral vowels; Florianopolis dialect; Children speech; Acoustic analysis

\section{Introdução}

O objetivo deste estudo é descrever acusticamente as sete vogais orais em posição tônica produzidas por crianças florianopolitanas de 10 e 11 anos de idade, antes da fase de muda vocal ${ }^{1}$. Do estudo das vogais [i, e, $\varepsilon$, a, $\supset, \mathrm{o}, \mathrm{u}]$, pretendemos oferecer uma descrição acústica com base nas medidas físicas de duração e frequência de formantes. A esses parâmetros estão relacionados os movimentos articulatórios de produção das vogais: as frequências do primeiro e segundo formantes estão associadas, respectivamente, aos movimentos de altura e anteriori-

\footnotetext{
* A pesquisa é certificada pelo Comitê de Ética da Universidade Federal de Santa Catarina sob o número 2057 (FR434924).

1 Na fase de muda vocal, há uma grande variação em frequência nos sons produzidos, dada as alterações da frequência fundamental, características dessa fase.
}

zação/recuo do corpo da língua (ISTRE, 1983; SEARA, 2008; CRISTÓFARO-SILVA, 2002, dentre outros).

Aos estudos acústicos que têm reportado aquilo que é universal entre os sistemas vocálicos das línguas, somamse as pesquisas que caracterizam aquilo que é particular de cada inventário, como por exemplo, Peterson e Barney (1952) para o inglês americano; Gordeeva et al. (2003) para o inglês escocês e o russo; Van Son e Pols (1990) para as vogais do holandês; Seara (2000) para o português brasileiro; Escudero et al. (2009) para as vogais do português brasileiro e europeu, para citar alguns. As especificidades metodológicas e os resultados dessas pesquisas determinam os avanços no estudo acústico das vogais, seja em direção à análise da qualidade acústica da vogal em diferentes estágios do desenvolvimento infantil, seja com relação ao padrão dessas vogais, se universal ou específico de uma determinada língua 
(LEE et al. 1999; BUSBY e PLANT 1995; BENNETT 1981; HILLEBRAND et al. 1995; ANDRADE, 2009; ESCUDERO et al. 2009; CUNHA, 2011).

Hillebrand et al. (1995), por exemplo, ao replicar o estudo de Peterson e Barney (1952), analisaram as medidas de frequência de F1, F2 e F3 das vogais [i, I, $\mathrm{e}, \varepsilon, \mathfrak{a}, \mathrm{a}, \supset, \mathrm{o}, \mathrm{v}, \mathrm{u}, \Lambda]$ do inglês americano produzidas por crianças com idades entre 10 e 12 anos. Embora os autores não tenham analisado separadamente dados de sujeitos masculinos e femininos, o espaço acústico F1-F2 das vogais das crianças mostrou uma tendência à centralização.

Considerando as diferenças entre os sexos, Busby e Plant (1995) investigaram as medidas de frequência dos três primeiros formantes das onze vogais do inglês australiano em sílaba tônica (/i/, /I/, / $/ \varepsilon /, / æ /, / a /, / \Lambda /$, $/ \mathrm{o} /, / \mathrm{p} /, / \mathrm{u} /, / \mathrm{v} /, / 3 /)$. Analisando a fala de 40 crianças, divididas nos grupos de cinco, sete, nove e onze anos de idade, os resultados obtidos pelos autores mostraram que as frequências dos três primeiros formantes vocálicos foram, em linhas gerais, mais elevadas para os sujeitos femininos. Além disso, as medidas de F1, F2 e F3 apresentaram uma redução em função da idade para todas as vogais analisadas, quanto mais velhos menores os valores dessas frequências. Especialmente para a faixa etária de 11 anos, as mudanças relacionadas à idade e sexo foram observadas para o primeiro formante das vogais $/ \mathfrak{x} /, / \mathrm{a} /, / \Lambda /$, sugerindo que $\mathrm{F} 1$ seja sensível às alterações relacionadas a esses dois fatores. De acordo com os autores, esse resultado pode ser atribuído às diferenças, em tamanho, das cavidades oral e faríngea uma vez que essas vogais seriam produzidas com maior abertura da cavidade oral e sem constrição no trato.

Os achados reportados por Busby e Plant (1995) ratificam as conclusões divulgadas por Bennett (1981). Em um estudo conduzido com 42 crianças com idades entre 7 e 8 anos, a autora analisou os valores de F1, F2, F3 e F4 de cinco vogais do inglês americano produzidas em um contexto fonético fixo (I will say $d^{*}$ d again) com o objetivo de avaliar as características dos formantes vocálicos em função do gênero. Os resultados obtidos mostraram que os valores de frequência das vogais analisadas foram mais elevados para informantes femininos. Para Bennett (1981), as diferenças nas frequências dos formantes das crianças estariam relacionadas às diferenças de tamanho da cavidade oral entre meninos e meninas.

Em um estudo mais extenso, Lee et al. (1999) analisaram as alterações nos parâmetros acústicos de duração e frequência dos formantes em função da idade e gênero a partir de dados obtidos de 436 crianças com idades entre 5 e 17 anos e 56 adultos entre 25 e 50 anos. As medidas foram coletadas com base na leitura de uma sentença-veículo e, para crianças de 5 e 6 anos, repetição de palavra isolada. Os autores observaram que as diferenças nos parâmetros de frequência formântica iniciam por volta dos 11 anos, tornando-se estáveis aos 15 . Durante esse período, foram observadas alterações mais lineares nos formantes vocálicos dos sujeitos masculinos, enquanto, para os informantes femininos, essa tendência foi menos aparente.

A exemplo de estudos sobre as frequências dos formantes vocálicos, estudos sobre a duração de vogais têm sugerido que diversos fatores podem influenciar nos seus resultados. Além do contexto de tonicidade, se tônico, pretônico ou átono final, a taxa de elocução, o vozeamento, a altura da vogal, e também aspectos sintáticos e semânticos, como a posição da palavra no enunciado ou a familiaridade com o vocábulo são fatores condicionantes. House e Fairbanks (1953), pesquisando sobre a influência do contexto consonantal e do vozeamento da consoante na duração das vogais, observaram que a duração das vogais foi superior em contextos vozeados se comparados aos não vozeados. Da mesma forma, em contexto de consoante fricativa, a duração das vogais analisadas foi superior se comparada aos demais modos de articulação, resultado que os autores atribuíram ao movimento gradual e controlado das consoantes contínuas.

Sobre o efeito da idade e sexo na duração das vogais, Lee et al. (1999) não observaram interação significativa em função do sexo, mas sim da idade. De acordo com os autores, enquanto a redução na duração das vogais dos 10 aos 12 anos e dos 11 aos 15 anos foi significativa, não foram observadas diferenças significativas na duração da vogal entre os grupos acima de 12 anos. Os resultados reportados pelos autores corroboram com o estudo de Hillebrand et al. (1995), que observaram diferenças significativas na duração das vogais entre os três grupos de sujeitos estudados (crianças de 10 e 12 anos e adultos).

A contribuição mais expressiva sobre a produção das vogais vem dos estudos em Fonoaudiologia que concentram sua investigação, sobretudo, na análise dos parâmetros de frequência fundamental, jitter e shimmer (BEHLAU, 2001; CAPELLARI et al. 2008; SADER e HANAYAMA 2004; VIEGAS et al. 2009). E mais recentemente, estudos acústicos sobre as vogais do $\mathrm{PB}$ e PE foram realizados por Moraes et al. (1996), Berti e Reato (2011) e Cunha (2011).

Berti e Reato (2011) compararam medidas acústicas de frequência formântica, duração e intensidade das vogais tônicas /i/, /a/ e /u/ do PB na fala de crianças com idades entre cinco e seis anos em dois experimentos: um de repetição e outro de conversa espontânea. Analisando os parâmetros acústicos em diferentes contextos fonéticos, as autoras não observaram diferenças significativas entre os resultados dos dois experimentos. Cunha (2011), ao investigar a variação acústica das vogais orais do PE com 
crianças de sete e dez anos de idade, observou que, aos 10 anos, sujeitos do sexo feminino apresentaram valores de formantes mais elevados do que sujeitos do sexo masculino da mesma faixa etária.

Ainda o padrão duracional das sete vogais tônicas do PB foi estudado por Hamel (1983) em experimentos de fala controlada realizados com três sujeitos adultos do sexo masculino. Com base nos resultados obtidos, os valores mais elevados de duração foram observados para as vogais médias baixas $[\varepsilon]$ e $[0]$ e para a vogal baixa central [a]; as vogais altas [i] e [e] apresentaram os valores mais baixos.

Como os estudos do PB e PE e de outras línguas que muito já avançaram na caracterização acústica das vogais do seu inventário, o presente estudo é constituído de um experimento com as sete vogais orais tônicas do português brasileiro (PB), quais sejam, as vogais [i, e, $\varepsilon, a, o, o, u]$, observando as mudanças acústicas relativas às frequências de ressonância e à duração relativa, comparando a produção de crianças do sexo masculino e feminino, todas florianopolitanas. Com a finalidade de detalhar um pouco mais as análises, foi feito um recorte, avaliando as vogais $[a, e, o]$ que se apresentaram em maior quantidade na base de dados pesquisada. Para apresentar a descrição dos resultados obtidos, este artigo se divide em mais três seções: na primeira apresentaremos a metodologia empregada para os experimentos e seus resultados; na seguinte, mostraremos os resultados obtidos nos experimentos aqui propostos e, na última, as considerações finais.

\section{Metodologia}

Para este estudo, foram selecionadas oito crianças, sendo quatro do sexo masculino e quatro do sexo feminino, com idades entre dez e onze anos, do ensino fundamental da rede pública da grande Florianópolis, todas florianopolitanas.

A amostra de fala infantil contemplou experimentos de produção realizados com base na leitura de dois corpora: o primeiro, composto de 92 palavras, entre dissílabas, trissílabas e polissílabas, paroxítonas e proparoxítonas, inseridas na sentença-veículo Digo * baixinho; e o segundo, com base na leitura de um texto no qual algumas das palavras do primeiro corpus foram inseridas. Foram coletadas todas as ocorrências das vogais orais $[\mathrm{e}, \mathrm{i}, \varepsilon, \rho$, $\mathrm{o}, \mathrm{u}, \mathrm{a}]$ em contexto tônico e pretônico. Dado o design dos experimentos, diferenças com relação ao número de dados obtidos para cada uma das vogais analisadas foram observadas, sendo superior para as vogais [e a o].

Para o conjunto das vogais $[\mathrm{e}, \mathrm{a}, \mathrm{o}]$, o contexto consonantal foi controlado a partir do ponto de articulação das consoantes que precediam as vogais, dados que foram organizados em três grupos: pré-alveolar (bilabial e labiodental), alveolar e pós-alveolar (palatal, velar e uvular). Esses grupos são compostos internamente por consoantes homorgânicas. Bhaskararao (1999) observou, para o desenvolvimento de sistemas de síntese de fala, que um único segmento transicional para uma vogal seguida de qualquer consoante oclusiva apresentando um mesmo ponto de articulação (SEARA et al. 2002) poderia ser usado para sintetizar diferentes segmentos dentro dessa mesma classe de homorgânicos ${ }^{2}$. Assim, acreditamos que os grupos formados darão conta das diferenças se elas existirem.

As vogais e as palavras que continham as vogais foram segmentadas e etiquetadas manualmente no software Praat 5.2.15. Para a segmentação, os seguintes critérios foram adotados: a duração das vogais foi determinada entre o primeiro e o último período regular da vogal; e, para palavras iniciadas por plosiva surda, a fase do silêncio da plosiva foi estabelecida em $40 \mathrm{~ms}^{3}$ iniciais até o VOT. Esse procedimento foi adotado para a extração de duração da palavra, necessária para a medida de duração relativa. Casos de repetição, ruído externo ou correção foram excluídos das análises por conta da qualidade da produção.

Para a análise acústica das vogais, as medidas de duração relativa e frequência do primeiro e segundo formantes das vogais foram extraídas automaticamente. Os valores de frequência dos formantes foram obtidos da porção estável da vogal e a duração relativa do segmentoalvo foi obtida a partir da relação entre a duração absoluta da vogal $(m s)$ e a duração absoluta da palavra $(m s)$. Tal estratégia foi utilizada para que se obtivesse o percentual de ocupação do segmento vocálico na palavra, eliminando diferenças relacionadas às taxas de elocução nas diferentes produções dos sujeitos da pesquisa.

A análise estatística foi realizada utilizando o programa estatístico SPSS ${ }^{4}$. Primeiramente, os valores dos parâmetros - duração relativa, frequências de F1, F2 não normalizadas, e frequências de F1 e F2 normalizadas - foram analisados para a distribuição normal (Gaussiana) e, como alguns dos grupos não apresentaram distribuição normal, testes não-paramétricos foram utilizados para verificar se havia ou não diferenças significativas entre os grupos. A partir do valor de $p$ (probabilidade de significância), foi observado se as diferenças eram ou não significativas: valores abaixo de 0,05 foram considerados significativos, e valores de $p$ acima de 0,05 foram considerados não significativos, com base em Barbetta (2011) e Martins (2011).

\footnotetext{
2 Homorgânicos são dois ou mais fonemas que têm um mesmo ponto de articulação, diferenciando-se por outros traços (DUBOIS, 1973). Por exemplo, [t] e [d] são homorgânicos.

3 Essa estratégia foi amparada em Seara (2000), em que foram obtidas medidas da fase de oclusão de consoantes oclusivas do PB e a média desses dados correspondeu a 40ms. Daí adotarmos esse valor neste estudo.

4 SPSS Statistic 17.0. Polar Engineering and Consulting, copyright 19932007
} 
Dois tipos de testes não paramétricos foram aplicados, um para comparações entre dois grupos e outro para comparações entre três ou mais grupos. Nos casos de comparação entre dois grupos, o Teste de Mann-Whitney foi utilizado, sendo considerados significativos os valores de $p$ abaixo de 0,05. Para comparar três ou mais grupos independentes, o Teste de Kruskal-Wallis (MARTINS, 2011) foi utilizado. Nos casos em que esse teste indicava haver diferenças significativas entre os grupos, foi aplicado, em seguida, o Teste de Mann-Whitney, entre cada par de grupos, para verificar onde estavam essas diferenças. Nesses casos, foi utilizada a Correção de Bonferroni ${ }^{5}$, de modo que o valor de referência passou a ser: $p=0,017$, quando havia 3 grupos.

Para a normalização dos dados, o método de Lobanov foi utilizado, o qual exclui diferenças fisiológicas nos valores dos formantes, mas mantém as diferenças sociolinguísticas. A fórmula para a normalização é a seguinte:

$$
\mathrm{Fn}[\mathrm{v}] \mathrm{N}=(\mathrm{Fn}[\mathrm{v}]-\operatorname{média}(\mathrm{n})) / \mathrm{DP}(\mathrm{n})
$$

onde: $F n[v] N$ é o valor normalizado para $F n[v]$ (isto é, para o formante $n$ da vogal $v$ ); média ( $n$ ) é o valor médio para o formante n para o falante em questão; e $D P(n)$ é o desvio padrão para o formante $n$ do falante.
Como todos os métodos de normalização apresentam vantagens e desvantagens, o método de Lobanov foi selecionado pela possibilidade de exclusão das diferenças fisiológicas e por sua facilidade de uso através do site: $<$ http://ncslaap.lib.ncsu.edu/tools/norm/norm1. php $>$. No endereço, é possível também obter os gráficos dos espaços acústicos com os dados normalizados.

\section{Resultados}

Iniciamos a apresentação dos resultados obtidos para os formantes das vogais [e, i, $\varepsilon, \jmath, o, u, a]$ em contexto tônico. Em seguida, a partir de testes estatísticos, apresentamos as diferenças relevantes referentes ao fator gênero (masculino-feminino).

Os resultados da coleta das frequências dos formantes F1 e F2, em dados não normalizados, das sete vogais orais investigadas são apresentados na Tab. 1 . Também são descritos os resultados referentes às diferenças estatísticas em função do gênero. Apresentamos os resultados estatísticos para dados não normalizados, uma vez que vários estudos discutidos na literatura não utilizaram o procedimento de normalização na análise de seus dados.

Tabela 1 - Comparações entre sexo feminino e masculino do grupo das crianças florianopolitanas, com relação às frequências de $\mathrm{F} 1$ e F2 das vogais $[\mathrm{i}, \mathrm{e}, \varepsilon, \mathrm{a}, \mathrm{0}, \mathrm{o}, \mathrm{u}]$, produzidas pelos sujeitos sem normalização.

\begin{tabular}{|c|c|c|c|c|c|c|c|}
\hline \multirow{3}{*}{$\begin{array}{c}\text { Frequência } \\
\mathrm{F} 1(\mathrm{~Hz})\end{array}$} & \multirow{3}{*}{$\begin{array}{c}\text { Vogal } \\
{[i]}\end{array}$} & \multicolumn{3}{|c|}{ Dados normalizados } & \multicolumn{3}{|c|}{ Dados não normalizados } \\
\hline & & \multirow{2}{*}{$\begin{array}{c}\text { Média } \\
(411-419)\end{array}$} & \multicolumn{2}{|c|}{ Teste* $^{*}$} & \multirow{2}{*}{$\begin{array}{c}\text { Média } \\
(335-330)\end{array}$} & \multicolumn{2}{|c|}{ Teste* $^{*}$} \\
\hline & & & $Z=-, 84$ & $p=, 401$ & & $Z=-, 52$ & $p=, 605$ \\
\hline & [e] & $(516-511)$ & $Z=-, 45$ & $p=, 655$ & $(378-383)$ & $Z=-1,36$ & $p=, 174$ \\
\hline & {$[\varepsilon]$} & (799-709) & $Z=-3,18$ & $p=, 001$ & $(495-499)$ & $Z=-, 40$ & $p=, 692$ \\
\hline & [a] & $(988-834)$ & $Z=-10,38$ & $p=, 000$ & $(573-572)$ & $Z=-, 03$ & $p=, 979$ \\
\hline & [0] & $(811-700)$ & $Z=-3,85$ & $p=, 000$ & $(500-494)$ & $Z=-, 88$ & $p=, 378$ \\
\hline & [o] & $(560-519)$ & $Z=-3,63$ & $p=, 000$ & $(396-388)$ & $Z=-1,77$ & $p=, 077$ \\
\hline & {$[\mathrm{u}]$} & $(442-456)$ & $z=-1,05$ & $p=, 294$ & $(348-351)$ & $Z=-, 85$ & $p=, 395$ \\
\hline \multirow[t]{7}{*}{$\mathrm{F} 2(\mathrm{~Hz})$} & [i] & $(2792-2606)$ & $Z=-4,45$ & $p=, 000$ & (1985-2005) & $Z=-1,62$ & $p=, 106$ \\
\hline & {$[e]$} & $(2501-2368)$ & $Z=-5,43$ & $p=, 000$ & $(1819-1850)$ & $Z=-, 48$ & $p=, 631$ \\
\hline & {$[\varepsilon]$} & $(2300-2141)$ & $Z=-2,67$ & $p=, 008$ & (1703-1703) & $Z=-, 71$ & $p=, 480$ \\
\hline & [a] & $(1820-1652)$ & $Z=-6,78$ & $p=, 000$ & $(1428-1385)$ & $Z=--3,64$ & $p=, 000$ \\
\hline & [0] & $(1369-1260)$ & $Z=-2,01$ & $p=, 045$ & $(1169-1131)$ & $Z=-1,50$ & $p=, 132$ \\
\hline & [o] & $(1331-1331)$ & $Z=-, 08$ & $p=, 936$ & $(1147-1176)$ & $Z=-, 67$ & $p=, 504$ \\
\hline & {$[\mathrm{u}]$} & $(1094-1262)$ & $Z=-1,55$ & $p=, 121$ & $(1011-1131)$ & $Z=-1,68$ & $p=, 093$ \\
\hline
\end{tabular}

* Teste de Mann-Whitney entre os grupos das crianças (feminino e masculino).

Legenda: $\mathrm{DP}=$ desvio padrão; $Z=$ valor do teste estatístico; $p=$ nível de significância; resultados significativos em negrito $(p<, 05)$.

\footnotetext{
5 A Correção de Bonferroni é utilizada para evitar que ocorram erros nos resultados significativos e consiste em dividir o valor do ponto de corte convencional relativo à significância $(p=0,05)$ pelo número de testes adicionais aplicados na sequência de um teste de diferenças significativo que compara três ou mais grupos (MARTINS, 2011).
}

Os resultados mostraram diferenças significativas para todas as vogais em análise, à exceção de F1 das vogais [i, e] e de $\mathrm{F} 2$ das vogais $[\mathrm{o}, \mathrm{u}]$ nas produções infantis florianopolitanas (Tab. 1). Essas diferenças, 
já evidenciadas na literatura (BENNETT, 1981; VORPERIAN e KENT, 2007; ANDRADE, 2009; ESCUDERO et al., 2009; CUNHA, 2011), mostraram que os valores médios dos formantes vocálicos foram mais altos para sujeitos do sexo feminino se comparados aos resultados obtidos para sujeitos do sexo masculino no grupo aqui analisado. Tais diferenças seriam atribuídas aos ajustes da cavidade faríngea, espessura de pregas vocais, grau de abertura da mandíbula e, ou, diferenças no tamanho da cavidade oral entre meninos e meninas (BENNETT, 1981; BUSBY et al., 1995; RAUBER, 2008; ESCUDERO et al., 2009; CUNHA, 2011).

Para viabilizar a comparação das vogais orais em posição tônica (nosso objeto de pesquisa) sem a interferência de diferenças fisiológicas, os dados foram normalizados, segundo os procedimentos e método mostrados na Seção 2, e estão apresentados na Tab. 1. Com relação a esses dados, os resultados mostraram que as diferenças estão relacionadas apenas ao movimento de anterioridade/recuo (referente a F2). Assim, podemos verificar que a vogal [a] parece ser a vogal mais afetada pelo fator sexo, sendo menos centralizada para as meninas, dado o valor elevado para F2. Essas observações corroboram os achados de Busby e Plant (1995) que observaram serem as vogais [a $\Lambda$ æ] as mais afetadas pelo mesmo fator. Para as demais vogais, não foram observadas diferenças estatisticamente relevantes.

Behlau (2001), pesquisando a fala infantil, observou valores médios das frequências das vogais anteriores mais baixos para as falantes femininas. Nossos dados não corroboram os achados dessa autora. Os dados não normalizados apresentaram vogais anteriores com valores médios significativamente mais altos (e, portanto, vogais mais anteriores) para as crianças do sexo feminino. Já os dados normalizados não mostraram diferenças significativas entre as vogais anteriores (Tab. 1). Talvez os diferentes critérios metodológicos adotados como a faixa etária investigada, gênero, tamanho da amostra e ferramenta de análise do sinal poderiam explicar as divergências aqui apontadas.
Os resultados encontrados em Bennett (1981) seguem a tendência observada na literatura geral: crianças do sexo feminino apresentaram valores de F1 superiores aos valores obtidos para crianças do sexo masculino, à exceção da vogal alta anterior, para as quais os sujeitos masculinos apresentaram valores mais altos. No presente estudo, em dados não normalizados, as vogais mostraram essa mesma tendência, à exceção das anteriores [i e] que não apresentaram diferenças significativas. Há divergências, no entanto, em relação aos resultados reportados por Hillebrand et al. (1995) que apresentou o comportamento anterior (valores mais altos para as crianças do sexo masculino), também com respeito às vogais altas anteriores.

$\mathrm{Se}$, porém, compararmos os dados normalizados com os resultados obtidos na literatura, veremos que, em relação ao movimento vertical de altura de língua (F1), não há diferenças significativas para nenhuma das vogais investigadas. Esses resultados nos parecem, portanto, mais confiáveis visto que subtraem a influência de fatores fisiológicos, como espessura de pregas vocais ou diferenças no tamanho da cavidade oral entre meninos e meninas.

Os resultados de Andrade (2009) são corroborados apenas pelos dados do grupo infantil sem normalização (Tab. 1) que apresentaram valores médios de F2 mais elevados para os sujeitos femininos, à exceção das vogais posteriores [o] e [u]. Porém, enquanto, para Andrade (2009), as vogais posteriores apresentaram valores de F2 mais baixos para sujeitos do sexo feminino, nossos dados não mostraram diferenças estatisticamente relevantes entre sexo para essas duas vogais no grupo infantil.

Considerando apenas as diferenças significativas para dados normalizados (Tab. 1), temos que, para as crianças, apenas a vogal baixa mostrou diferenças significativas entre os sexos, sendo mais centralizada para falantes do sexo masculino. Assim, o padrão apresentado pelos sujeitos masculinos denota uma tendência à centralização das vogais, já observada para adultos masculinos em diversos estudos (LEE et al., 1999; RAUBER, 2008). Vejamos a disposição dessas vogais no espaço acústico F1-F2 para cada grupo de sujeitos aqui pesquisados (Fig. 1).
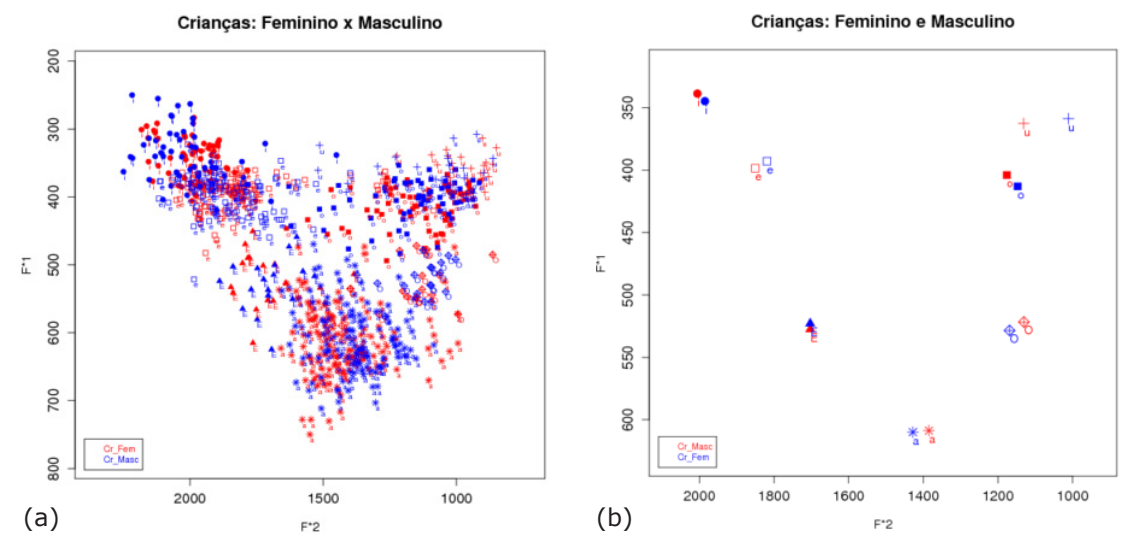

Figura 1 - Gráficos do espaço acústico (F1xF2) dos valores de: (a) dispersão e (b) dos valores médios dos formantes das vogais orais em posição tônica para sujeitos infantis do sexo masculino (azul) e feminino (vermelho). 
Tabela 2 - Valores médios e desvio padrão da duração relativa (\%) das vogais [i, e, $\varepsilon, \mathrm{a}, \mathrm{o}, \mathrm{o}, \mathrm{u}]$ produzidas pelas crianças do sexo masculino e do feminino e comparação entre esses grupos para cada vogal em separado

\begin{tabular}{ccccccc}
\hline \multirow{2}{*}{ Duração } & Vogal & \multicolumn{2}{c}{ Feminino } & \multicolumn{2}{c}{ Masculino } & \multicolumn{2}{c}{$\begin{array}{c}\text { Teste* } \\
\text { Média (DP) }\end{array}$} & $\begin{array}{c}\text { Número } \\
\text { de dados }\end{array}$ & Média (DP) & $\begin{array}{c}\text { Número } \\
\text { de dados }\end{array}$ & (Feminino x Masculino) \\
\hline Relativa & {$[\mathrm{i}]$} & $17,82(8,31)$ & 57 & $17,61(7,64)$ & 51 & $\mathrm{Z}=-1,33, p=, 184$ \\
& {$[\mathrm{e}]$} & $18,70(7,91)$ & 94 & $20,00(5,39)$ & 93 & $\mathrm{Z}=-, 47, p=, 637$ \\
& {$[\varepsilon]$} & $22,29(9,48)$ & 17 & $24,94(8,03)$ & 17 & $\mathrm{Z}=-, 38, p=, 704$ \\
& {$[\mathrm{a}]$} & $23,85(8,70)$ & 129 & $25,87(6,49)$ & 127 & $\mathrm{Z}=-1,10, p=, 273$ \\
& {$[0]$} & $24,37(6,35)$ & 19 & $25,28(3,83)$ & 18 & $\mathrm{Z}=-, 03, p=, 976$ \\
& {$[\mathrm{o}]$} & $18,92(8,29)$ & 61 & $20,98(7,46)$ & 60 & $\mathrm{Z}=-, 99, p=, 324$ \\
& {$[\mathrm{u}]$} & $16,63(6,23)$ & 24 & $14,52(5,35)$ & 25 & $\mathrm{Z}=-1,50, p=, 135$ \\
\hline
\end{tabular}

* Teste de Mann-Whitney entre os grupos (feminino $x$ masculino).

Legenda: $\mathrm{DP}=$ desvio padrão; $\mathrm{Z}=$ valor do teste estatístico; $p=$ nível de significância; resultados significativos em negrito $(p<, 05)$.

Do conjunto de parâmetros avaliados, os resultados para a análise de duração relativa (\%) das vogais orais do PB em contexto tônico são resumidos na Tab. 2. Os resultados obtidos mostraram não haver diferenças significativas entre os dois grupos analisados, crianças do sexo feminino e masculino. Porém, indicaram um movimento ascendente, das vogais altas em direção à vogal baixa.

Agora, para verificarmos se havia diferenças significativas entre os valores de duração relativa das vogais $[i, e, \varepsilon, a, \rho, o, u]$ entre si, em cada um dos grupos de crianças, aplicamos o Teste de Kruskal-Wallis. Os resultados foram significativos tanto para o grupo feminino $\left(\chi^{2}(6)=57,01, p=, 000\right)$ quanto para o masculino $\left(\chi^{2}(6)=96,64, p=, 000\right)$. Aplicando-se o Teste de MannWhitney entre cada par de vogais, observamos onde estavam as diferenças significativas. As Tabelas 3 e 4 mostram os resultados das comparações dos valores de duração relativa cruzando as vogais $[i, e, \varepsilon, a, o, o, u]$ produzidas pelas crianças do sexo feminino e masculino, respectivamente. Essas tabelas trazem somente os resultados das comparações que foram significativas.

Esses resultados mostram que, para as crianças do sexo feminino, a vogal baixa [a] difere em sua duração relativa quando comparada às vogais altas e médias altas (Tab. 3). A vogal média-baixa posterior [0] difere em relação às vogais anteriores alta [i] e média-alta [e] e em relação à alta posterior [u]; e a média-baixa anterior [ع] não apresenta diferenças em relação a nenhuma outra vogal. As diferenças apresentadas para as vogais [a] e [0] para o sexo feminino se repetem nos dados de sujeitos masculinos. Porém, para a vogal média-baixa anterior $[\varepsilon]$, há diferenças em relação à vogal alta posterior [u]; e esta última apresenta diferenças significativas em relação à vogal baixa [a], e em relação a todas as vogais médias (Tab. 4). Assim, o movimento ascendente observado no padrão duracional é parcialmente confirmado para os

Tabela 3 - Comparações dos valores da duração relativa (entre parênteses) cruzando as vogais [i, e, $\varepsilon$, a, o, o, u] produzidas pelas crianças do sexo feminino

\begin{tabular}{|c|c|c|c|c|c|c|c|c|}
\hline \multirow[t]{2}{*}{ Vogais } & \multicolumn{2}{|r|}{ [i] } & [e] & \multirow{2}{*}{$\begin{array}{c}{[\varepsilon]} \\
(22,29)\end{array}$} & \multirow{2}{*}{$\begin{array}{c}\text { [a] } \\
(23,85)\end{array}$} & \multirow{2}{*}{$\begin{array}{c}\text { [0] } \\
(24,37)\end{array}$} & \multirow{2}{*}{$\begin{array}{c}\text { [0] } \\
(18,92)\end{array}$} & \multirow{2}{*}{$\frac{[u]}{(16,63)}$} \\
\hline & Dur. relativa & $(17,82)$ & $(18,70)$ & & & & & \\
\hline [i] & $(17,82)$ & n.s & n.s & n.s & $\begin{array}{c}Z=-5,06 \\
p=, 000\end{array}$ & $\begin{array}{c}Z=-3,62 \\
p=, 000\end{array}$ & n.s & n.s \\
\hline [e] & $(18,70)$ & n.s & n.s & n.s & $\begin{array}{c}Z=-4,95 \\
p=, 000\end{array}$ & $\begin{array}{c}Z=-3,43 \\
p=, 001\end{array}$ & n.s & n.s \\
\hline$[\varepsilon]$ & $(22,29)$ & n.s & n.s & n.s & n.s & n.s & n.s & n.s \\
\hline [a] & $(23,85)$ & $\begin{array}{c}Z=-5,06 \\
p=, 000\end{array}$ & $\begin{array}{c}Z=-4,95 \\
p=, 000\end{array}$ & n.s & n.s & n.s & $\begin{array}{c}Z=-4,187 \\
p=, 000\end{array}$ & $\begin{array}{c}Z=-4,59, \\
p=, 000\end{array}$ \\
\hline [0] & $(24,37)$ & $\begin{array}{c}Z=-3,62 \\
p=, 000\end{array}$ & $\begin{array}{c}Z=-3,43 \\
p=, 001\end{array}$ & n.s & n.s & n.s & n.s & $\begin{array}{c}Z=-4,32, \\
p=, 000\end{array}$ \\
\hline [o] & $(18,92)$ & n.s & n.s & n.s & $\begin{array}{c}Z=-4,187 \\
p=, 000\end{array}$ & n.s & n.s & n.s \\
\hline$[\mathbf{u}]$ & $(16,63)$ & n.s & n.s & n.s & $\begin{array}{c}Z=-4,59 \\
p=, 000\end{array}$ & $\begin{array}{c}Z=-4,32 \\
p=, 000\end{array}$ & n.s & n.s \\
\hline
\end{tabular}

* Teste de Mann-Whitney cruzando as vogais $[i, e, \varepsilon, a, o, o, u]$ produzidas pelas crianças do sexo feminino.

Legenda: n.s. = não significativo. $Z=$ valor do teste estatístico; $p=$ probabilidade de significância, resultados significativos $(p<, 002)$. 
Tabela 4 - Comparações dos valores da duração relativa (entre parênteses) entre as vogais [i, e, $\varepsilon, a, 0,0, u]$ produzidas pelas crianças do sexo masculino

\begin{tabular}{|c|c|c|c|c|c|c|c|c|}
\hline \multirow[t]{2}{*}{ Vogais } & & [i] & [e] & {$[\varepsilon]$} & [a] & [0] & [o] & [u] \\
\hline & Dur. rel. & $(17,61)$ & $(20,00)$ & $(24,94)$ & $(25,82)$ & $(25,28)$ & $(20,98)$ & $(14,52)$ \\
\hline [i] & $(17,61)$ & n.s. & n.s. & n.s. & $\begin{array}{c}Z=-6,32 \\
p=, 000\end{array}$ & $\begin{array}{c}Z=-4,11 \\
p=, 000\end{array}$ & n.s. & n.s. \\
\hline [e] & $(20,00)$ & n.s. & n.s. & n.s. & $\begin{array}{c}Z=-6,62 \\
p=, 000\end{array}$ & $\begin{array}{c}Z=-3,86 \\
p=, 000\end{array}$ & n.s. & $\begin{aligned} Z & =-3,91, \\
p & =, 000\end{aligned}$ \\
\hline$[\varepsilon]$ & $(24,94)$ & n.s. & n.s. & n.s. & n.s. & n.s. & n.s. & $\begin{aligned} Z & =-3,93, \\
p & =, 000\end{aligned}$ \\
\hline [a] & $(25,82)$ & $\begin{array}{c}Z=-6,32, \\
p=, 000\end{array}$ & $\begin{array}{c}Z=-6,62, \\
p=, 000\end{array}$ & n.s. & n.s. & n.s. & $\begin{aligned} Z & =-4,31 \\
p & =, 000\end{aligned}$ & $\begin{aligned} Z & =-6,67, \\
p & =, 000\end{aligned}$ \\
\hline [0] & $(25,28)$ & $\begin{array}{c}Z=-4,11 \\
p=, 000\end{array}$ & $\begin{array}{c}Z=-3,86 \\
p=, 000\end{array}$ & n.s. & n.s. & n.s. & n.s. & $\begin{aligned} Z & =-5,05, \\
p & =, 000\end{aligned}$ \\
\hline [o] & $(20,98)$ & n.s. & n.s. & n.s. & $\begin{array}{c}\mathrm{Z}=-4,31 \\
p=, 000\end{array}$ & n.s. & n.s. & $\begin{aligned} Z & =-3,57, \\
p & =, 000\end{aligned}$ \\
\hline [u] & $(14,52)$ & n.s. & $\begin{array}{c}Z=-3,91 \\
p=, 000\end{array}$ & $\begin{array}{c}Z=-3,93 \\
p=, 000\end{array}$ & $\begin{array}{c}Z=-6,67 \\
p=, 000\end{array}$ & $\begin{array}{c}Z=-5,05 \\
p=, 000\end{array}$ & $\begin{array}{c}Z=-3,57 \\
p=, 000\end{array}$ & n.s. \\
\hline
\end{tabular}

* Teste de Mann-Whitney cruzando as vogais $[i, e, \varepsilon, a, o, o, u]$ produzidas pelas crianças do sexo masculino.

Legenda: n.s. = não significativo. $Z=$ valor do teste estatístico; $p=$ probabilidade de significância; resultados significativos $(p<, 002)$.

dados de sujeitos masculinos, que exibem diferenças significativas entre as altas posteriores e as médias posteriores. A diferença de duração relativa entre a vogal baixa e as altas e médias-altas anteriores e posteriores também se mostrou consistente, nesse caso, para os dois sexos.

$\mathrm{Na}$ variação observada nos dados de duração relativa, referentes às vogais $[i, e, \varepsilon, a, o, o, u]$, o teste de Mann-Whitney revelou que os grupos das crianças do sexo feminino e do masculino não apresentam diferenças significativas entre si, conforme indicam os resultados da Tabela 2. Esses resultados em função do sexo diferem dos resultados reportados por Cunha (2011) que verificou valores de duração superiores para vogais produzidas por crianças do sexo feminino. Lembramos, no entanto, que Cunha (2011) apresentou dados de duração absoluta, assim a diferença apontada por essa autora poderia estar associada às diferentes taxas de elocução dos sujeitos.

No presente estudo, as diferenças de duração relativa verificada entre as categorias vocálicas estariam relacionadas aos movimentos articulatórios produzidos para a realização das vogais. As vogais baixas, por exemplo, exigem um tempo maior de produção, se comparado às outras vogais. Essa diferença em tempo foi considerada relevante para os dois sexos. De acordo com House e Fairbanks (1953), a duração das vogais estaria diretamente relacionada ao grau de abertura da cavidade oral e inversamente relacionada à altura da língua. Assim, vogais baixas apresentariam maior duração em relação às vogais altas, dada a extensão do movimento articulatório necessário para a produção da vogal baixa e, consequentemente, o tempo correspondente (LEHISTE, 1970; HAMEL, 1983). Essas diferenças entre vogais baixas e altas foram estatisticamente confirmadas.
Agora, passemos à análise das vogais [e, a, o], verificando diferenças relacionadas aos fatores tonicidade e ponto de articulação da consoante precedente. Conforme descrito na Seção 2, o tamanho da amostra não permitiu estender essa análise para as demais vogais, dado o design dos experimentos.

\subsection{O recorte das vogais orais $[e, a, o]$}

Para análise do efeito de fatores contexto de tonicidade e ponto de articulação, o conjunto das vogais orais [e, a, o] em posição tônica e pré-tônica foi selecionado. Nesta seção, são apresentados e discutidos os resultados obtidos para análise dos parâmetros acústicos frequência dos formantes e duração, considerando o sexo das crianças em separado. Os testes estatísticos foram rodados sobre dados normalizados. Vejamos os resultados na seção a seguir.

\subsubsection{Tonicidade}

$\mathrm{Na}$ Tab. 5, referente à tonicidade das sílabas em que se encontram as vogais produzidas pelas crianças florianopolitanas, observamos que houve diferenças estatisticamente significativas entre os valores das frequências normalizadas de F1 e F2.

Com referência a F1, os resultados do Teste de MannWhitney mostraram que a vogal [a] apresenta valores mais altos na posição tônica do que na pré-tônica, tanto no grupo das crianças do sexo feminino quanto no do sexo masculino (Tab. 5). A vogal [e], no entanto, mostrou frequências significativamente mais baixas na posição tônica somente para as produções do grupo masculino. 
Tabela 5 - Valores médios e desvio padrão das frequências de $\mathrm{F} 1$ e $\mathrm{F} 2(\mathrm{~Hz})$ das vogais [e, a, o], com relação às posições pré-tônica e tônica, produzidas pelas crianças do sexo feminino (fem.) e masculino (mas.) - dados normalizados

\begin{tabular}{|c|c|c|c|c|c|c|c|}
\hline \multirow[b]{2}{*}{ Frequência } & \multirow[b]{2}{*}{ Sujeitos } & \multirow[b]{2}{*}{ Vogal } & \multicolumn{2}{|c|}{ Pré-tônica } & \multicolumn{2}{|c|}{ Tônica } & \multirow[b]{2}{*}{$\begin{array}{c}\text { Teste }^{*} \\
\text { (Pré-tônica x Tônica) }\end{array}$} \\
\hline & & & Média (DP) & $\begin{array}{l}\text { Número } \\
\text { dados }\end{array}$ & Média (DP) & $\begin{array}{l}\text { Número } \\
\text { de dados }\end{array}$ & \\
\hline \multirow[t]{6}{*}{$\mathrm{F} 1(\mathrm{~Hz})$} & Fem. & [e] & $356(25)$ & 115 & $355926)$ & 94 & $Z=-, 863, p=, 388$ \\
\hline & & [a] & $504(61)$ & 190 & $595(57)$ & 129 & $Z=-11,13, p=, 000$ \\
\hline & & [o] & $364(29)$ & 90 & 377 (32) & 61 & $Z=-2,08, p=, 038$ \\
\hline & Mas. & [e] & $361(34)$ & 127 & $352(33)$ & 93 & $Z=-2,53, p=, 011$ \\
\hline & & [a] & $520(55)$ & 189 & $582(53)$ & 127 & $Z=-8,80, p=, 000$ \\
\hline & & [o] & $363(38)$ & 83 & $358(32)$ & 60 & $Z=-1,29, p=, 199$ \\
\hline \multirow[t]{6}{*}{$\mathrm{F} 2(\mathrm{~Hz})$} & Fem. & {$[\mathrm{e}]$} & $1780(241)$ & 115 & $1928(216)$ & 94 & $Z=-5,99, p=, 000$ \\
\hline & & [a] & $14879184)$ & 190 & $1483(169)$ & 129 & $Z=-, 59, p=, 556$ \\
\hline & & [o] & $11919225)$ & 90 & $1164(188)$ & 61 & $Z=-, 383, p=, 702$ \\
\hline & Mas. & {$[\mathrm{e}]$} & $1836(185)$ & 127 & $1944(158)$ & 93 & $Z=-4,38, p=, 000$ \\
\hline & & [a] & $1463(158)$ & 189 & $1410(138)$ & 127 & $Z=-2,90, p=, 004$ \\
\hline & & [o] & $11839220)$ & 83 & $1170(222)$ & 60 & $Z=-, 483, p=, 629$ \\
\hline
\end{tabular}

* Teste de Mann-Whitney entre as posições (pré-tônica e tônica).

Legenda: $\mathrm{DP}=$ desvio padrão; $Z=$ valor do teste estatístico; $p=$ nível de significância; resultados significativos em negrito $(p<, 05)$.

A vogal [o] apresentou valores mais elevados na posição tônica apenas nas produções do grupo feminino, corroborando o comportamento da vogal [a]. As demais comparações não apresentaram diferenças significativas. Com respeito a F2, a vogal [e] apresentou os valores mais elevados na posição tônica, tanto para o grupo feminino quanto para o masculino. Também foram encontradas diferenças significativas para a vogal [a], produzida pelo grupo masculino, que apresentou, no entanto, valores mais altos na posição pré-tônica.

Os resultados computados para os grupos em análise indicaram uma tendência à centralização das vogais no contexto pré-tônico se comparado ao contexto tônico. Esse padrão reforça a disposição à centralização de vogais pré-tônicas do PB já observada por Nobre e Ingeman (1983) na fala de adultos.

Observando cada vogal em separado, considerando apenas a diferença entre os contextos de tonicidade, percebemos que há diferenças significativas para as três vogais em análise (Tab. 6). Os resultados mostram que as vogais em sílaba tônica apresentam-se sistematicamente mais longas do que em posição pré-tônica, corroborando os resultados apresentados por Nobre e Ingeman (1983) para falantes adultos.
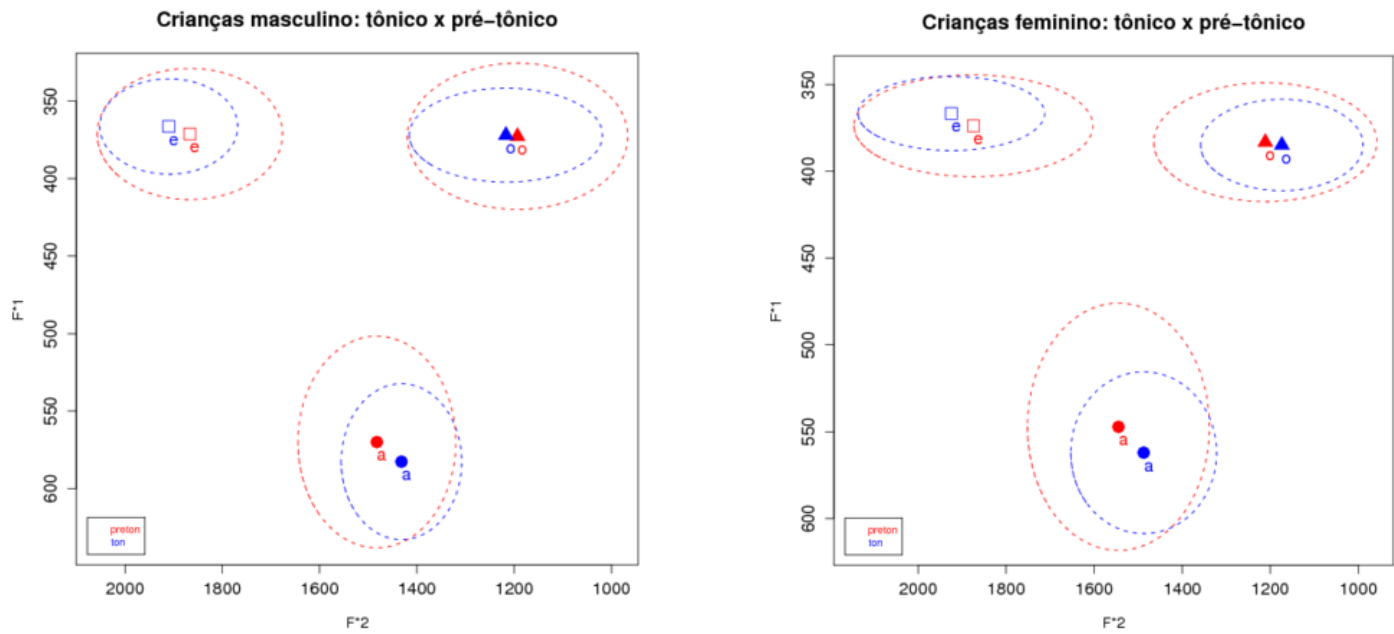

Figura 2 - Média dos dois primeiros formantes vocálicos (F1 e F2) e elipses representando a dispersão em torno da média das produções infantis do sexo masculino e feminino, considerando-se o contexto de tonicidade: pré-tônico (vermelho) e tônico (azul). 
Tabela 6 - Valores médios e desvio padrão da duração relativa (\%) das vogais [e, a, o], em posição pré-tônica e tônica, referente às crianças do sexo masculino e do sexo feminino e comparação entre essas posições.

\begin{tabular}{|c|c|c|c|c|c|c|}
\hline \multirow[b]{2}{*}{ Sujeitos } & \multirow[b]{2}{*}{ Vogal } & \multicolumn{2}{|c|}{ Pré-tônica } & \multicolumn{2}{|c|}{ Tônica } & \multirow{2}{*}{$\begin{array}{c}\text { Teste }^{*} \\
\text { (Pré-tônica x Tônica) }\end{array}$} \\
\hline & & Média (DP) & $\begin{array}{l}\text { Número } \\
\text { dados }\end{array}$ & Média (DP) & $\begin{array}{l}\text { Número } \\
\text { de dados }\end{array}$ & \\
\hline \multirow[t]{3}{*}{ Feminino } & [e] & $14,07(6,57)$ & 115 & $18,70(7,91)$ & 94 & $Z=-16,64, p=, 000$ \\
\hline & [a] & $15,11(6,67)$ & 190 & $23,85(8,70)$ & 129 & $Z=-9,71, p=, 000$ \\
\hline & [o] & $13,06(5,70)$ & 90 & $18,92(8,29)$ & 61 & $Z=-4,80, p=, 000$ \\
\hline \multirow[t]{3}{*}{ Masculino } & {$[\mathrm{e}]$} & $16,69(6,25)$ & 127 & $20,00(5,39)$ & 93 & $Z=-4,11, p=, 000$ \\
\hline & [a] & $16,49(5,34)$ & 188 & $25,87(6,49)$ & 127 & $Z=-11,54, p=, 000$ \\
\hline & [o] & $14,13(6,26)$ & 83 & $20,98(7,46)$ & 60 & $Z=-5,36, p=, 000$ \\
\hline
\end{tabular}

* Teste de Mann-Whitney entre as posições (pré-tônica $x$ tônica).

Legenda: $\mathrm{DP}=$ desvio padrão; $Z$ = valor do teste estatístico; $p=$ nível de significância; resultados significativos em negrito $(p<, 05)$.

\subsubsection{Ponto de articulação}

As vogais médias-altas anterior [e] e posterior [o] e a vogal baixa central [a] em ambos os contextos de tonicidade foram analisadas em função do ponto de articulação para o grupo infantil florianopolitano, observando as diferenças para cada sexo separadamente. Os resultados são apresentados na Fig. 3 e Tab. 7.
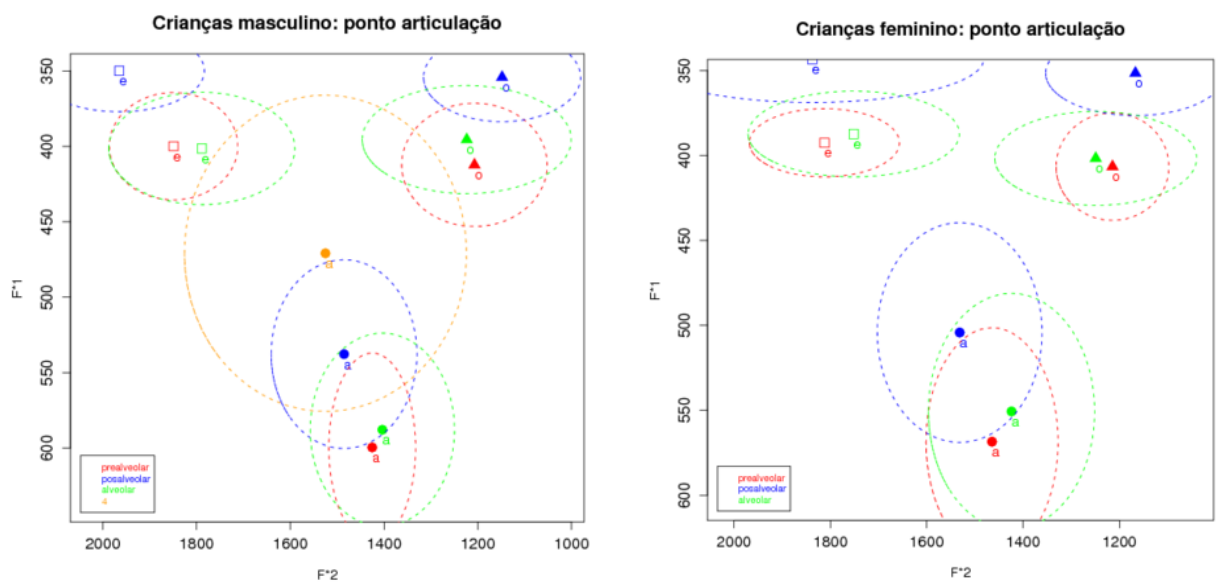

Figura 3 - Média dos dois primeiros formantes vocálicos (F1 e F2) e elipses representando a dispersão em torno da média das produções infantis do sexo masculino e feminino, considerando-se o ponto de articulação: pré-alveolar (vermelho), pós-alveolar (azul) e alveolar (verde).

Tabela 7 - Valores médios e desvio padrão das frequências normalizadas de F1 e F2 (Hz) das vogais [e, a, o], com relação aos contextos pré-alveolar, alveolar e pós-alveolar, produzidas pelas crianças do sexo feminino e masculino e comparação entre esses pontos de articulação.

\begin{tabular}{|c|c|c|c|c|c|c|c|c|c|}
\hline \multirow{2}{*}{ Freq. } & \multirow{2}{*}{ Sujeitos } & \multirow{2}{*}{ Vogal } & \multicolumn{2}{|c|}{ Pré-alveolar } & \multicolumn{2}{|c|}{ Alveolar } & \multicolumn{2}{|c|}{ Pós-alveolar } & \multirow{2}{*}{$\begin{array}{c}\text { Teste* }^{*} \\
\text { (Pré-alv. x Alv x Pós-alv.) }\end{array}$} \\
\hline & & & Média (DP) & N. dados & Média (DP) & N. dados & Média (DP) & N. dados & \\
\hline \multirow[t]{6}{*}{$\mathrm{F} 1(\mathrm{~Hz})$} & Fem. & [e] & $353(21)$ & 80 & $356(27)$ & 96 & $359(30)$ & 33 & $\chi^{2}(2)=3,25, p=, 197$ \\
\hline & & [a] & $538(71)$ & 72 & $534(75)$ & 96 & $546(75)$ & 151 & $\chi^{2}(2)=2,56, p=, 278$ \\
\hline & & [o] & 367 (33) & 65 & $372(30)$ & 50 & $368(29)$ & 36 & $\chi^{2}(2)=, 89, p=, 642$ \\
\hline & Mas. & [e] & $354(34)$ & 81 & $362(35)$ & 99 & $353(29)$ & 40 & $\chi^{2}(2)=2,60, p=, 272$ \\
\hline & & [a] & $543(59)$ & 72 & $538(60)$ & 91 & $554(67)$ & 130 & $\chi^{2}(2)=5,94, p=, 051$ \\
\hline & & [o] & 366 (39) & 60 & $356(34)$ & 50 & $358(31)$ & 33 & $\chi^{2}(2)=1,70, p=, 427$ \\
\hline \multirow[t]{6}{*}{$\mathrm{F} 2(\mathrm{~Hz})$} & Fem. & [e] & $1864(204)$ & 80 & $1845(227)$ & 96 & 1807 (348) & 33 & $\chi^{2}(2)=, 53, p=, 768$ \\
\hline & & [a] & $1410(179)$ & 72 & 1507 (178) & 96 & $1508(167)$ & 151 & $\chi^{2}(2)=21,94, p=, 000$ \\
\hline & & [o] & $1083(153)$ & 65 & $1326(217)$ & 50 & $1152(182)$ & 36 & $\chi^{2}(2)=36,54, p=, 000$ \\
\hline & Mas. & [e] & $1889(171)$ & 81 & 1857 (189) & 99 & 1928 (179) & 40 & $\chi^{2}(2)=5,73, p=, 057$ \\
\hline & & [a] & $1360(116)$ & 72 & $1491(146)$ & 91 & $1457(153)$ & 130 & $\chi^{2}(2)=34,52, p=, 000$ \\
\hline & & [o] & $1087(194)$ & 60 & $1320(212)$ & 50 & $1125(165)$ & 33 & $\chi^{2}(2)=39,65, \boldsymbol{p}=, 000$ \\
\hline
\end{tabular}

* Teste de Kruskal-Wallis entre os contextos (pré-alveolar, alveolar e pós-alveolar)

Legenda: $\mathrm{DP}=$ desvio padrão; $\chi^{2}=$ valor do teste estatístico; $p=$ nível de significância; resultados significativos em negrito $(p<, 05)$. 
Considerando as diferenças relacionadas ao ponto de articulação das consoantes que precedem as vogais em análise, pré-alveolar, alveolar e pós-alveolar, observamos diferenças significativas para o segundo formante, conforme mostra a Tab. 7. Os dados referentes a F2 das vogais [a] e [o] mostraram diferenças com relação aos pontos de articulação, tanto para o grupo das crianças do sexo feminino quanto para as do sexo masculino. Através do Teste Mann-Whitney, pôde-se identificar onde se encontravam essas diferenças.

Com relação aos valores de F2 da vogal [a], do grupo feminino, o contexto pré-alveolar diferenciou-se significativamente dos demais contextos, apresentando valores mais baixos do que os contextos alveolar $(\mathrm{Z}=-4,11, p=, 000)$ e pós-alveolar $(\mathrm{Z}=-4,31, p=, 000)$. Já, com relação à vogal $[\mathrm{o}]$, o contexto alveolar diferenciouse significativamente dos demais contextos, apresentando valores de F2 maiores do que os apresentados para os contextos pré-alveolar $(\mathrm{Z}=-5,85, p=, 000)$ e pós-alveolar $(\mathrm{Z}=-3,76, p=, 000)$.

O grupo masculino apresentou resultados semelhantes: para a vogal [a], os valores de F2 foram mais altos no contexto pré-alveolar do que nos contextos alveolar $(\mathrm{Z}=-5,76, p=000)$ e pós-alveolar $(\mathrm{Z}=-4,37, p=, 000)$; e, para a vogal [o], os valores de F2 foram mais altos no contexto alveolar do que nos contextos pré-alveolar $(\mathrm{Z}=-6,01, p=, 000)$ e pós-alveolar $(\mathrm{Z}=-4,25, p=, 000)$.

A duração relativa foi igualmente mensurada em função do ponto de articulação da consoante precedente para o conjunto das vogais orais $[\mathrm{e}, \mathrm{a}, \mathrm{o}]$. Os resultados obtidos são apresentados na Tab. 8.

A comparação entre os valores de duração relativa, considerando-se os pontos de articulação pré-alveolar, alveolar e pós-alveolar das consoantes que precedem as vogais (Tab. 8), mostrou que a vogal [o] exibe diferenças significativas, tanto para o grupo feminino quanto para o masculino. Aplicando-se o Teste de Mann-Whitney, pudemos identificar que os grupos feminino e masculino apresentam duração da vogal [o] significativamente maior na posição pós-alveolar do que nas posições préalveolar $(\mathrm{Z}=-2,51, p=, 012$, feminino; $\mathrm{Z}=-3,81, p=, 000$, masculino) e alveolar $(Z=-3,22, p=, 001$, feminino; $\mathrm{Z}=-3,83, p=, 000$, masculino). As posições pré-alveolar e alveolar não apresentaram diferenças significativas $(p>, 05)$ entre si.

\section{Considerações finais}

Propusemos, neste estudo, uma descrição acústica das sete vogais orais tônicas do português brasileiro produzidas por crianças florianopolitanas de 10 e 11 anos de idade com base em F1, F2 e duração relativa.

Restringindo as diferenças fisiológicas e considerando apenas as diferenças sociolinguísticas, verificamos que o sistema vocálico de crianças florianopolitanas do sexo masculino e feminino não apresentou diferenças significativas na qualidade das vogais - a exceção estaria apenas na vogal baixa, menos centralizada para crianças do sexo feminino.

Em relação à duração relativa, verificamos que não houve diferenças entre crianças do sexo feminino e masculino. As diferenças observadas estariam relacionadas aos movimentos articulatórios produzidos para a realização das vogais. Assim, as vogais baixas necessitam de um tempo maior de produção, se comparada às outras vogais. Essa diferença em tempo foi considerada relevante para os dois sexos.

Investindo, sobretudo, na normalização de dados, acreditamos que a facilidade de uso das metodologias atualmente disponíveis $<$ http://ncslaap.lib.ncsu.edu/tools/ norm/norm1.php $>$, certamente levará outros pesquisadores a analisarem seus dados com foco mais centrado em características linguísticas, isentas das diferenças fisiológicas, como o que se buscou com o presente estudo.

Tabela 8 - Valores médios e desvio padrão da duração relativa (\%) das vogais $[e, a, o]$, com relação aos contextos pré-alveolar, alveolar e pós-alveolar, referentes às crianças do sexo feminino (fem.) e do sexo masculino (mas.), e comparação entre esses contextos

\begin{tabular}{|c|c|c|c|c|c|c|c|c|}
\hline \multirow{2}{*}{ Sujeitos } & \multirow{2}{*}{ Vogal } & \multicolumn{2}{|c|}{ Pré-alveolar } & \multicolumn{2}{|c|}{ Alveolar } & \multicolumn{2}{|c|}{ Pós-alveolar } & \multirow{2}{*}{$\begin{array}{c}\text { Teste }^{*} \\
\text { (Pré-alv. x Alv x Pós-alv.) }\end{array}$} \\
\hline & & Média (DP) & N. dados & Média (DP) & N. dados & Média (DP) & N. dados & \\
\hline \multirow[t]{3}{*}{ Fem. } & {$[e]$} & $16,90(7,29)$ & 80 & $15,45(7,82)$ & 96 & $16,39(7,40)$ & 33 & $\chi^{2}(2)=1,01, p=, 603$ \\
\hline & [a] & $18,42(7,11)$ & 72 & $18,80(9,02)$ & 96 & $18,65(9,18)$ & 151 & $\chi^{2}(2)=, 04, p=, 980$ \\
\hline & [o] & $15,11(7,73)$ & 65 & $13,50(5,89)$ & 50 & $18,67(7,87)$ & 36 & $\chi^{2}(2)=11,08, p=, 004$ \\
\hline \multirow[t]{3}{*}{ Mas. } & {$[\mathrm{e}]$} & $18,19(6,16)$ & 81 & $18,78(5,69)$ & 99 & $16,18(6,75)$ & 40 & $\chi^{2}(2)=4,09, p=, 130$ \\
\hline & [a] & $19,73(6,56)$ & 72 & $20,30(8,04)$ & 91 & $20,52(7,46)$ & 153 & $\chi^{2}(2)=, 357, p=, 837$ \\
\hline & [o] & $15,68(6,43)$ & 60 & $15,36(7,37)$ & 50 & $21,91(7,90)$ & 33 & $\chi^{2}(2)=18,69, p=, 000$ \\
\hline
\end{tabular}

* Teste de Kruskal-Wallis entre os contextos (pré-alveolar, alveolar e pós-alveolar).

Legenda: DP=desvio padrão; $\chi^{2}=$ valor do teste estatístico; $p=$ nível de significância; resultados significativos em negrito $(p<, 05)$. 


\section{Referências}

ANDRADE, Flávia. V. de. Análise de parâmetros espectrais da voz em crianças saudáveis de 4 a 8 anos. 2009. 61 fls. Dissertação (Mestrado em Fonoaudiologia) - Universidade Veiga de Almeida, Rio de Janeiro, 2009.

BARBETTA, Pedro. A. Estatística Aplicada às Ciências Sociais. Florianópolis: Editora da UFSC, 2011.

BEHLAU, Mara. Voz: o livro do especialista. Rio de Janeiro : REVINTER, 2001. v. 1.

BENNETT, Suzanne. Vowel formant frequency characteristics of preadolescent males and females. Journal of the Acoustic Society of America, v. 69, n. 1, 1981.

BERTI, Larissa C.; REATO, Laiz. T. Comparação entre medidas acústicas de vogais em provas de repetição e conversa espontânea. Linguagem \& Ensino, Pelotas, v. 14, n. 2, p. 553572, jul./dez. 2011.

BUSBY, Peter. A.; PLANT, G. L. Formant frequency values of vowels produced by preadolescent boys and girls. Journal of the Acoustic Society of America, v. 97, n. 4, 1995.

CAPELLARI, Viviane. M. de; CIELO, Carla. A. Caracteristicas vocais acústicas de crianças pré-escolares. Revista Brasileira de Otorrinolaringologia, v. 74, n. 2, p. 265-272, 2008.

CUNHA, Maria. M. M. de O. Variação acústica das vogais orais de crianças do português europeu. 2011. 47fls. Dissertação (Mestrado em Ciências da Fala e da Audição) - Universidade de Aveiro, Portugal, 2011.

GORDEEVA, Olga; MENNEN, Ineke; SCOBBIE, James M. Vowel duration and spectral balance in Scottish English and Russian. Proceedings of the 15th International Congress of Phonetic Sciences. Barcelona, 2003.

ESCUDERO, Paola; BOERSMA, Paul; RAUBER, Andréia S.; BION, Ricardo. A cross-dialect acoustic description of vowels: Brazilian and European Portuguese. Journal of the Acoustic Society of America, v. 126, n. 3, 2009.

HAMEL, Patricia. J. Brazilian Portuguese stressed vowels: a durational study. Kansas Working Papers in Linguistics, v. 8, n. 1, p. 31-46, 1983.

HILLEBRAND, James; GETTY, Laura A.; CLARK, Michael J.; WHEELER, Kimberlee. Acoustic characteristics of American English vowels. Journal of the Acoustic Society of America, v. 97, n. 5,1995

HOUSE, Arthur S.; FAIRBANKS, Grant. The influence of consonant environment upon the secondary characteristics of vowels. Journal of the Acoustic Society of America, v. 22, n. 457,1953 .

ISTRE, Giles. Fonologia transformacional e natural: uma introdução crítica. Florianópolis: NEL/SC, 1983. p. 37-72.
LEE, Sungbok; POTAMIANOS, Alexandros; NARAYANAN, Shrikanth. Acoustics of children's speech: developmental changes of temporal and spectral parameters. Journal of the Acoustic Society of America, v. 105, n. 3, 1999.

MARTINS, Carla. Manual de análise de dados quantitativos com recurso ao IBM SPSS. Braga: Psiquilibríos Edições, 2011.

MORAES, João. A.; CALLOU, Dinah.; LEITE, Yonne. O sistema vocálico do português do Brasil: caracterização acústica. In: KATO, Mary (Ed.). Gramática do Português Falado 5. Campinas: Editora da Unicamp, 1996. p. 33-53.

NOBRE, Maria. A.; INGEMAN, Frances. Oral vowel reduction in Brazilian Portuguese. Kansas Working Papers in Linguistics, v. 8, n. 1, p. $1-30,1983$.

PETERSON, Gordon. E.; BARNEY, Harold. L. Control methods used in a study of the vowels. Journal of the Acoustic Society of America, v. 24, 1952.

RAUBER, Andréia. An acoustic description of Brazilian Portuguese oral vowels. Diacrítica, Ciências da linguagem, v. 22, n. 1, p. 229-238, 2008

SEARA, Izabel.C.; SEARA, Rui; KAFKA, Sandra; KLEIN, Simone G.; PACHECO, Fernando S. Utilização de segmentos transicionais homorgânicos em síntese de fala concatenativa. In: CBA 2002 - CONGRESSO BRASILEIRO DE AUTOMÁTICA, XIV, 2002, Natal-RN. Anais do CBA 2002. Natal, 2002. p. 2742-2747.

SEARA, Izabel. C. et al. Fonética e fonologia do português brasileiro. Florianópolis: LLV/CCE/UFSC, 2008.

SEARA, Izabel. C. Estudo acústico-perceptual da nasalidade das vogais do português brasileiro. 2000. 271 fls. Tese (Doutorado) - Universidade Federal de Santa Catarina, Centro de Comunicação e Expressão, Florianópolis, 2000.

VAN SON, Rob. J. J. H.; POLS, L. C. W. Formant frequencies of Dutch vowels in a text, read at normal and fast rate. Journal of the Acoustic Society of America, v. 88, 1990.

VIEGAS, Flávia et al. Análise de parâmetros espectrais clássicos da voz em crianças respiradoras orais de 5 a 10 anos. 2009, 61 p. Dissertação (Mestrado) - Universidade Veiga de Almeida, Mestrado em Fonoaudiologia, Rio de Janeiro, 2009.

VORPERIAN, Houri. K.; KENT, Ray. D. Vowel acoustic space deveopment in children: a synthesis of acoustic and anatomic data. Journal of Speech, Language, and Hearing Research, v. 50, p. 1510-1545, 2007.

Recebido: 15 de novembro de 2012 Aprovado: 10 de janeiro de 2013 Contato: lilianminikel@gmail.com izabels@linse.ufsc.br 